\title{
GnRH agonist in association with hCG versus hCG alone for final oocyte maturation triggering in $\mathrm{GnRH}$ antagonist cycles
}

\author{
Condesmar M. de Oliveira Filho ${ }^{1}$, Carlos A. M. de Oliveira ${ }^{1}$, Larissa L. Fonseca ${ }^{1}$, Kelly R. R. de Souza ${ }^{1}$, Moacir \\ R.M. Radaelli \\ ${ }^{1}$ Núcleo Santista de Reprodução Humana, Santos - SP, Brazil \\ 2Urology Department, Medical School, Faculdade Ingá, Maringá, Brazil
}

\begin{abstract}
Objective: To analyze gonadotropin-releasing hormone $(\mathrm{GnRH})$ agonist in association with human chorionic gonadotropin (hCG) (dual triggering) versus hCG alone (conventional triggering) for final oocyte maturation triggering in $\mathrm{GnRH}$ antagonist cycles in an unselected population of Brazilian women.

Methods: This prospective case-control study involved 114 patients referred to autologous in vitro fertilization treatment between February 2018 and August 2019, recruited regardless of age, infertility factor or number of cycles. The patients were randomly allocated into two groups according to oocyte maturation triggering approach: group A $(n=48)$ - hCG only; and group B $(n=66)$ - hCG plus $\mathrm{GnRH}$ agonist. The main outcomes measured were the number of total and metaphase II (MII) oocytes retrieved.

Results: The groups were homogenous in terms of age. There were no moderate or severe ovarian hyperstimulation syndrome events. There were no statistical differences concerning total or MII oocytes retrieved between the groups $(p>0.05)$. The MII/total oocyte rate was $70.9 \%$ in group $\mathrm{A}$, and $74.5 \%$ in group $\mathrm{B}(p=0.679)$. There was no oocyte retrieved in $2 / 48$ patients $(4.16 \%)$ in group $A$, $1 / 66(1.5 \%)$ in group $B$. There were no MII oocytes in $4 / 48$ patients $(8.3 \%)$ in group $A$, and $2 / 66(3 \%)$ in group $B$. Age was directly correlated to the number of total and MII oocytes retrieved $(p<0.05)$.

Conclusions: Dual triggering was equivalent to conventional hCH triggering in terms of the number of total and MII oocytes retrieved in the general population. Further studies are necessary to ascertain dual triggering indication in selected groups of women.
\end{abstract}

Keywords: Oocyte, dual triggering, gonadotropinreleasing hormone, GnRH agonist, GnRH antagonist, human chorionic gonadotropin

\section{INTRODUCTION}

Controlled ovarian hyperstimulation $(\mathrm{COH})$ is one of the most important strategies involved in successful in vitro fertilization-embryo transfers (IVF-ET) (Penzias, 2004). At the end of $\mathrm{COH}$, human chorionic gonadotropin (hCG) is usually used as a surrogate $\mathrm{LH}$ surge to trigger the last stage of oocyte maturation, so that they can be retrieved and fertilized (Ludwig et al., 2003). However, some studies have suggested that hCG can have a negative impact on endometrial receptivity (Simon et al., 1995;1998; Forman et al., 1998) and embryo quality (Valbuena et al., 2001; Tavaniotou et al., 2002). Moreover, hCG has also been associated with increased risks of ovarian hyperstimulation syndrome (OHSS) in gonadotropin-releasing hormone $(\mathrm{GnRH})$ antagonist IVF cycles (Delvigne \& Rozenberg, 2003).
In $\mathrm{COH}$ regimens with $\mathrm{GnRH}$ antagonists to downregulate the cycle, $\mathrm{GnRH}$ agonists can be used alternatively to hCG to trigger the release of endogenous luteinizing hormone (LH) (Gonen et al., 1990; Tay, 2002). In this approach, the pituitary remains responsive to the $\mathrm{GnRH}$ agonist (Felberbaum et al., 1995; Orvieto et al., 2006), very similar to what happens in natural cycles (Nevo et al., 2003; Kol, 2004). Among the advantages of using $\mathrm{GnRH}$ agonists compared to hCG, we find the reduced risk of OHSS (Kol, 2004), a more physiological LH and FSH surge, which may result in improved oocyte (Humaidan et al., 2005) and endometrial quality (Forman et al., 1998; Simon et al., 1998). Nevertheless, a recent Cochrane analysis indicated that GnRH agonists alone should not be routinely used for final oocyte maturation, due to lower live birth rates and lower ongoing pregnancy rates (Youssef et al., 2014), which have been attributed to defective luteal phase function (Engmann \& Benadiva, 2010).

Recently, a novel strategy to modify ovulation triggering through the use of a bolus of GnRH agonist plus a reduced or standard dosage of hCG (dual trigger) was proposed (Shapiro et al., 2011; Griffin et al., 2014; Jung et al., 2014; Kim et al., 2014; O'Neill et al., 2016). The rationale behind this strategy was that triggering with a GnRH agonist would effectively minimize the risk of OHSS, while the added hCG would also preserve adequate luteal function. Moreover, it has been suggested that the dual trigger approach can also result in better oocyte maturation, blastulation and pregnancy rates (Shapiro et al., 2011; Lin et al., 2013; Kim et al., 2014; Orvieto, 2015).

However, whether the addition of hCG on the day of $\mathrm{GnRH}$ agonist triggering actually produces better oocyte retrieval outcomes remains controversial. In an attempt to elucidate this question, two systematic reviews and meta-analyses were recently conducted (Ding et al., 2017; Chen et al., 2018). In both cases, although the authors indicated that dual triggering seemed to be more favorable at improving pregnancy rates, it was equivalent to the hCG triggering in terms of the number of oocytes and mature oocytes retrieved. Because mature oocytes are a prerequisite in IVF cycles, further studies are required to better elucidate the most effective protocol.

Therefore, the objective of the present study was to analyze $\mathrm{GnRH}$ agonist in association with hCG (dual triggering) versus hCG alone (conventional triggering) for final oocyte maturation triggering in $\mathrm{GnRH}$ antagonist

cycles in a population of unselected Brazilian women.

\section{MATERIALS AND METHODS}

\section{Study population}

All patients admitted at a private fertility clinic in the city of Santos (Brazil) for autologous in vitro fertilization (IVF) treatment between February 2018 and August 2019, were recruited regardless of age, infertility factor (male 
factor, unexplained infertility, tubal factor infertility) or number of cycles (first or second).

The study was conducted in compliance with the ethical standards of Resolution 466/2012 of the Brazilian National Health Council, the 1964 Helsinki declaration and its later amendments, and the recommendations set by the Strengthening the Reporting of Observational Studies in Epidemiology (STROBE) guidelines (von Elm et al., 2014).

The study was approved by the local Institutional Review Board, and all participating patients signed an informed consent before participating in the study.

\section{Study design}

In this case-control prospective study, all participating patients followed the same $\mathrm{COH}$ using the $\mathrm{GnRH}$ antagonist protocol. At the moment of oocyte maturation triggering, patients were randomly allocated into two groups: group A (control group) - conventional hCG triggering; and group B (test group) GnRH agonist in association with hCG (dual triggering).

\section{Ovarian stimulation and triggering}

Ovarian stimulation for all patients followed the same in-house protocol, always conducted by the same doctor with large experience in assisted reproductive technology (ART). A daily dose of urinary FSH (Fostimon, UCB) 300 UI was administered from day 2 of the menstruation. GnRH antagonist (Orgalutran, MSD) $0.25 \mathrm{mg} / \mathrm{day}$ was administered from the moment follicles reached $15 \mathrm{~mm}$ in diameter until triggering. Follicular growth was monitored with transvaginal ultrasound scans every other day until triggering criteria were met, always by the same ultrasound specialist.

Final oocyte maturation was triggered when at least two follicles measuring $\geq 17 \mathrm{~mm}$ were observed. Patients allocated to group $A$, underwent conventional triggering with two boluses of hCG (Choriomon, UCB) 5,000 UI. Patients allocated to group $B$ underwent dual triggering with the administration of $1 \mathrm{ml}$ of $5 \mathrm{mg}$ leuprolide acetate (Lupron, Depot) and two boluses of hCG 5,000 UI.

All oocyte retrievals took place under transvaginal ultrasound guidance, 34-36 h after triggering, and harvested oocytes were sent to the embryology laboratory, where they were counted and classified according to the criteria established by Veeck (2002), always by the same experienced embryologist.

\section{Outcome variables}

The outcome variables of this study were the number of total and metaphase II (MII) oocytes retrieved.

\section{Statistical analysis}

Data collected were statistically analyzed with the assistance of Statistica 13.2 single user (TIBCO Statistica ${ }^{\circledR}$
- Palo Alto, CA, USA). Considering the non-parametric distribution of data, the groups were compared using the Mann-Whitney test. We used the Spearman correlation test to check the correlation between the variables (total number of oocytes, MII oocytes, and patients' age). The level of statistical significance was set at $p \leq 0.05$.

\section{RESULTS}

A total of 117 patients, aged between 21 and 38 years were included in the study period. A total of 50 patients were allocated to group $\mathrm{A}$ (conventional $\mathrm{hCH}$ triggering) and 67 patients to group $B$ (dual triggering). Two patients in group $A$ and one patient in group B had their cycles interrupted due to the absence of adequate number of follicles before triggering, and were removed from the analysis. There were no moderate or severe OHSS events in any of the patients.

The two groups were homogeneous concerning the age of patients $(p=0.824)$. No statistically significant differences in the number of total ( $p=0.604)$, or MII, oocytes $(p=0.502)$ retrieved were seen between the groups (Table 1 ). The MII/total oocyte rate was $70.9 \%$ in group $A$, and $74.5 \%$ in group $B$, with no statistically significant differences between the groups $(p=0.679)$. Only $2 / 48$ patients $(4.16 \%)$ had no oocyte retrieval in group $A$, when compared to $1 / 66$ patients $(1.5 \%)$ in group $B$ (Figure 1$)$. A total of $4 / 48$ patients $(8.3 \%)$ in group $A$ and $2 / 66(3 \%)$ in group B presented no MII oocyte retrieval (Figure 2).

Age was found to be positively correlated with both the number of total and MII oocytes retrieved $(p<0.05)$ (Table 2). As the patients got older the number of oocytes decreased, regardless of the triggering protocol (Figures 1 and 2).

\section{DISCUSSION}

This study was designed to check whether any advantage in terms of increasing the total number of oocytes and MII oocytes retrieval could be obtained with the use of the dual trigger approach when compared to the conventional hCG triggering in an unselected population of Brazilian women submitted to $\mathrm{COH}$ regimens with $\mathrm{GnRH}$ antagonist. Although the results demonstrated some benefits with the use of dual trigger, there were no statistically differences between the two strategies in terms of the number of total and MII oocytes retrieved.

A possible benefit of oocyte triggering using $\mathrm{GnRH}$ agonists is the more physiological approach, which stimulates both mid-cycle $\mathrm{LH}$ and FSH surge, similarly to what occurs in an ovulating healthy woman (Prochazka et al., 2012). However, studies comparing conventional $\mathrm{hCH}$ trigger with the dual trigger approach have reported controversial results on the outcome of final oocyte

Table 1. Mean, standard deviation ( $($ SD), minimum and maximum values for age, total oocytes and MII oocytes obtained for group A (hCG alone) and group B (hCG+GnRH agonist).

\begin{tabular}{|c|c|c|c|c|c|c|c|c|}
\hline Group & Variable & $\mathbf{n}$ & Mean & \pm & SD & Minimum & Maximum & $p^{*}$ \\
\hline A & \multirow{2}{*}{ Age (years) } & 48 & 33.1 & \pm & 3.9 & 23 & 38 & \multirow{2}{*}{0.8248} \\
\hline B & & 66 & 33.0 & \pm & 3.8 & 21 & 38 & \\
\hline A & \multirow{2}{*}{ Total oocytes (n) } & 48 & 10.2 & \pm & 8.5 & 0 & 38 & \multirow{2}{*}{0.6041} \\
\hline B & & 66 & 10.1 & \pm & 6.9 & 0 & 38 & \\
\hline A & \multirow{2}{*}{ MII oocytes (n) } & 48 & 7.2 & \pm & 6.1 & 0 & 24 & \multirow{2}{*}{0.5022} \\
\hline B & & 66 & 7.5 & \pm & 5.4 & 0 & 24 & \\
\hline
\end{tabular}

*Mann-Whitney test. 

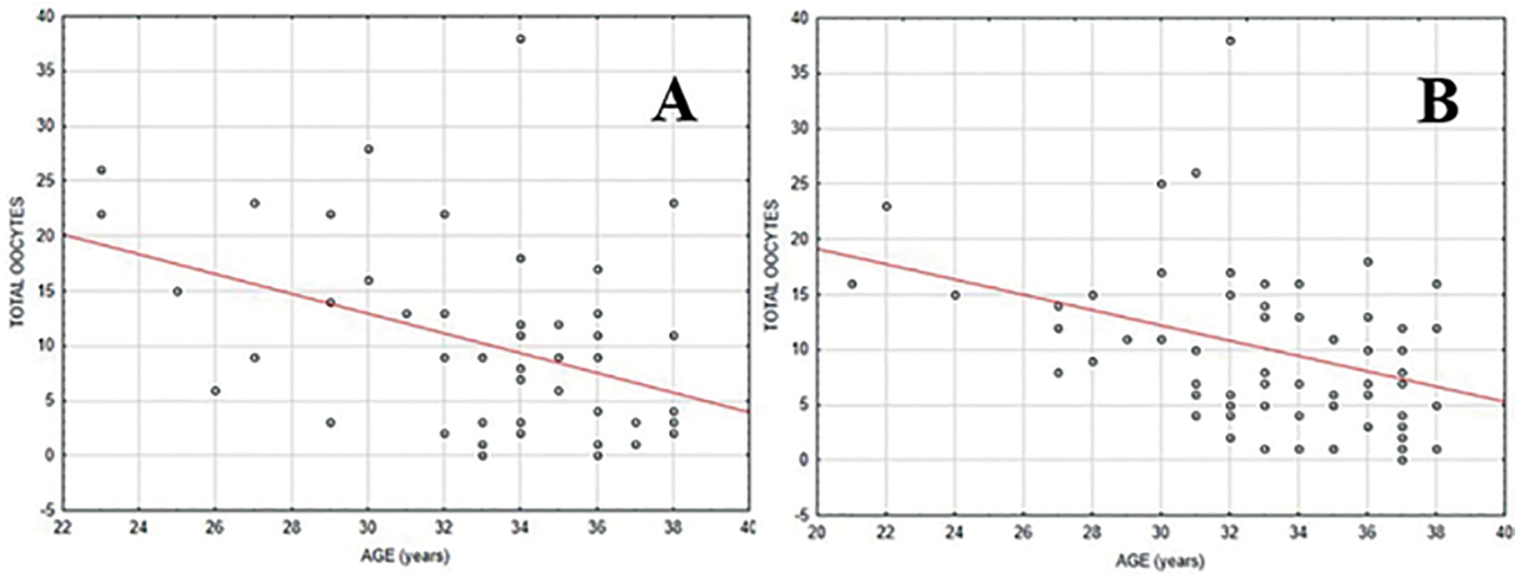

Figure 1. Distribution of the total number of oocytes retrieved in relation to the age of patients. A conventional trigger (hCG); B - dual trigger (hCG + GnRH agonist)
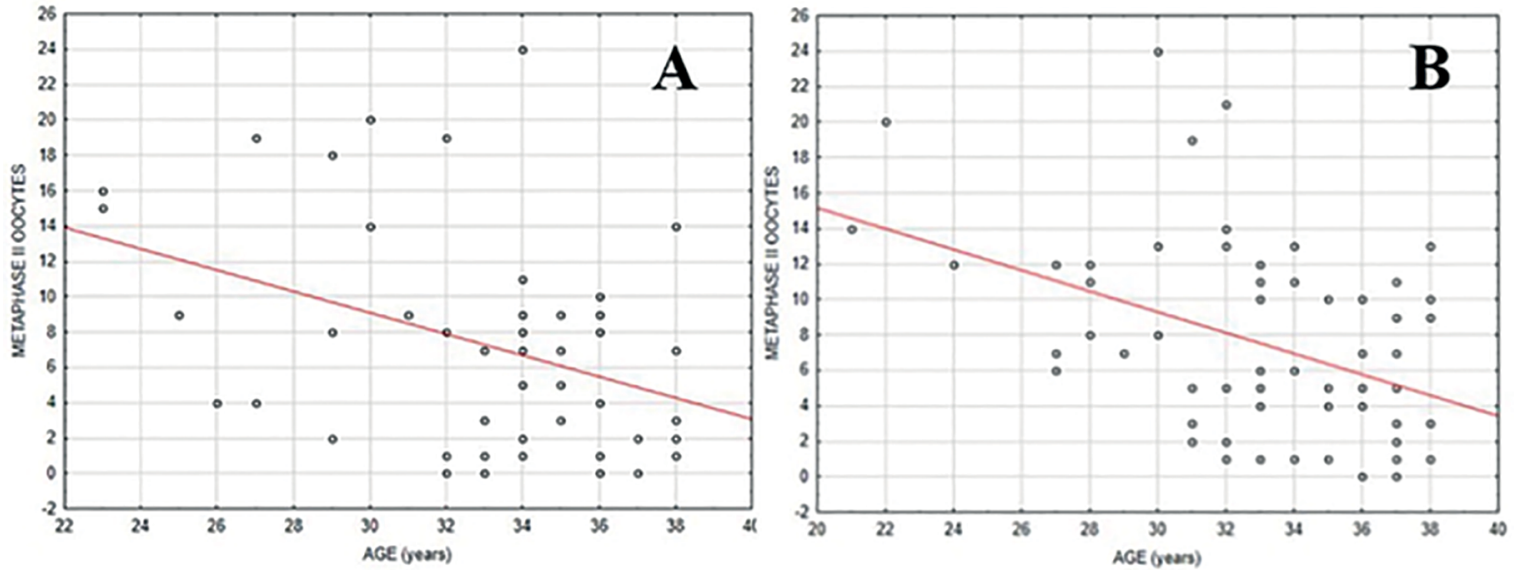

Figure 2. Distribution of metaphase II oocytes retrieved in relation to the age of patients. A - conventional trigger (hCG); B - dual trigger (hCG + GnRH agonist)

Table 2. Correlation among the studied variables.

\begin{tabular}{|l|c|c|}
\hline Variables & Age & Total oocytes \\
\hline Total oocytes & $-0.3844 *$ & $-\cdots$ \\
\hline Metaphase II oocytes & $-0.3514 *$ & 0.9590 \\
\hline
\end{tabular}

*Spearman correlation $(p<0.05)$.

maturation. Retrospective studies have shown that the number of total and mature oocytes retrieved were at least similar, and sometimes significantly higher with dual trigger (Lin et al., 2013; Lu et al., 2016; Seval et al., 2016, Jones et al., 2019). In a retrospective case-control study, Seval et al. (2016) demonstrated that although the mean number of total oocytes retrieved were similar, the mean number of metaphase II oocytes was significantly higher in the dual trigger group than in the $\mathrm{hCH}$ group. According to the authors, the higher number of MII oocytes observed with the dual trigger strategy was associated with the positive effect of FSH on pre-ovulatory follicles.

The findings of the present study seem to corroborate the perception that both strategies produce similar results in unselected patients. A non-significantly higher number of MII oocytes was retrieved with the dual trigger strategy, when compared with hCG alone. This finding is in agreement with previous prospective randomized controlled trials (RCTs), which also demonstrated that although the number of mature oocytes retrieved was higher with the dual trigger strategy than hCG alone, the difference did not reach statistical significance (Decleer et al., 2014; Kim et al., 2014; Mahajan et al., 2016). The same was also shown for the number of total oocytes retrieved (Schachter et al., 2008; Mahajan et al., 2016). When the data from these 4 RCTs were pooled together in two recent systematic reviews, the lack of significance between the groups in relation to the number of total and MII oocytes retrieved was confirmed (Ding et al., 2017; Chen et al., 2018).

Nonetheless, despite the lack of significance between groups, the use of dual triggering seemed to produce some benefits when compared to hCG alone. For instance, the mean MII/total oocyte rate was slightly higher with 
the dual trigger approach (74.5\%), when compared with hCG triggering alone (70.9\%). Moreover, there was also a smaller number of women with no oocytes retrieved after dual triggering, with just one patient $(1.5 \%)$, than in the hCG group, with 2 patients $(4.16 \%)$. The same trend was also seen for the number of women with no MII oocytes retrieved $(3.0 \% \times 8.3 \%)$. Although the findings of the present prospective study indicate that the dual trigger strategy may produce some slightly better results than $\mathrm{hCH}$ alone, they also confirm the lack of statistical significance between the strategies to retrieve fertilizable oocytes in unselected women undergoing $\mathrm{GnRH}$ antagonist IVF cycles. Figures 1 and 2 show that the total number and MII oocytes retrieved followed the same pattern regardless of the triggering approach used. Rather than the triggering protocol, age was the main factor to affect the number of oocytes retrieved, demonstrating statistical significance.

While this study indicated no improved number of retrieved oocytes obtained with the dual trigger protocol in the studied population, the approach may have an indication for patients suffering from specific conditions such as repetitive implantation failure (RIF), empty follicular syndrome, or previous poor oocyte-embryo quality (Kol \& Humaidan, 2010). In a study by Pacheco et al. (2014), the authors suggested that dual triggering could be helpful in retrieving mature oocytes from small follicles in poor responders. Additionally, Ben-Haroush et al. (2019) recently suggested that dual triggering should be considered when oocyte maturation rates after conventional hCG triggering is lower than $70 \%$. On the other hand, although dual triggering resulted in a significantly higher mean number of total oocytes and MII oocytes retrieved, it has not been recommended as the primary ovulation trigger in normal and high responders undergoing donation regimens due to the significantly higher rates of OHSS (Jones et al., 2019).

Some important points concerning the present study should be considered when the results are analyzed. This study was composed by all ART women referred to our service during the study period that accepted to participate in the study, regardless of age, infertility factor or number of cycles. While this sample selection approach presents the advantage of producing more generalizable findings throughout the population, it fails in accounting for more specific cases. Moreover, hCG doses used for final oocyte triggering can range from 1,500 to 2,500 IU in normo-responders, and 10,000 IU in poor-responders or in patients with hypothalamic amenorrhea (Jones et al., 2019). In the present study, a standard hCG dose of 10,000 IU was used in all patients regardless of oocyte response or trigger strategy, in order to reduce medication dose bias. Although, an increased risk of OHSS has been associated with hCG triggering (Delvigne \& Rozenberg, 2003), no patient in the present study presented any signs of moderate or severe OHSS.

\section{CONCLUSION}

Considering the results and the limitations of the present study, we can conclude that the dual trigger approach for final oocyte maturation is equivalent to conventional $\mathrm{hCH}$ triggering in terms of the number of total and MII oocytes retrieved in the general population. Further studies are necessary to ascertain dual triggering indication in selected groups of women.

\section{ACKNOWLEDGEMENTS}

The authors would like to thank Mr. Antonio Carlos Correa for his support with the English version of the paper.

\section{Disclosure statement}

The authors reported no potential conflict of interest

\section{Corresponding Author:}

Moacir Rafael Martins Radaelli

Urology Department

Medical School

Faculdade Ingá

Maringá, PR, Brazil.

Email: rafaradaelli@yahoo.com.br

\section{REFERENCES}

Ben-Haroush A, Sapir O, Salman L, Altman E, Garor R, Margalit T, Shufaro $Y$, Oron G. Does 'dual trigger' increase oocyte maturation rate? J Obstet Gynaecol. 2019;40:860-2. PMID: 31790320 DOI: 10.1080/01443615.2019.1674791

Chen $\mathrm{CH}$, Tzeng $\mathrm{CR}$, Wang $\mathrm{PH}$, Liu WM, Chang $\mathrm{HY}$, Chen $\mathrm{HH}$, Chen $\mathrm{CH}$. Dual triggering with $\mathrm{GnRH}$ agonist plus hCG versus triggering with hCG alone for IVF/ICSI outcome in GnRH antagonist cycles: a systematic review and meta-analysis. Arch Gynecol Obstet. 2018;298:17-26. PMID: 29600322 DOI: $10.1007 / s 00404-018-4751-3$

Decleer W, Osmanagaoglu K, Seynhave B, Kolibianakis S, Tarlatzis B, Devroey P. Comparison of hCG triggering versus hCG in combination with a GnRH agonist: a prospective randomized controlled trial. Facts Views Vis Obgyn. 2014;6:203-9. PMID: 25593695

Delvigne A, Rozenberg S. Review of clinical course and treatment of ovarian hyperstimulation syndrome. Hum Reprod Update. 2003;9:77-96. PMID: 12638783 DOI: $10.1093 /$ humupd/dmg005

Ding N, Liu X, Jian Q, Liang Z, Wang F. Dual trigger of final oocyte maturation with a combination of $\mathrm{GnRH}$ agonist and hCG versus a hCG alone trigger in $\mathrm{GnRH}$ antagonist cycle for in vitro fertilization: a systematic review and Meta-analysis. Eur J Obstet Gynecol Reprod Biol. 2017;218:92-8. PMID: 28957685 DOI: 10.1016/j.ejogrb.2017.09.004

Engmann L, Benadiva C. Ovarian hyperstimulation syndrome prevention strategies: luteal support strategies to optimize pregnancy success in cycles with gonadotropinreleasing hormone agonist ovulatory trigger. Semin Reprod Med. 2010;28:506-12. PMID: 21082510 DOI: 10.1055/s0030-1265678

Felberbaum PE, Reissmannb T, Kiipkera W, Bauera O, Al Hasania S, Diedrich C, Diedricha K. Preserved pituitary response under ovarian stimulation with HMG and GnRH antagonists (cetrorelix) in women with tubal infertility. Eur J Obstet Gynecol Reprod Biol. 1995;61:151-5. PMID: 7556837 DOI: 10.1016/0301-2115(95)02138-W

Forman R, Fries N, Tastart J, Belaisch J, Hazout A, Frydman R. Evidence of an adverse effect of elevated serum estradiol concentrations on embryo implantation. Fertil Steril. 1998;49:118-22. PMID: 3335258 DOI: 10.1016/S00150282(16)59661-7

Gonen Y, Balakier H, Powell W, Casper RF. Use of gonadotropin- releasing hormone agonist to trigger follicular maturation for in vitro fertilization. J Clin Endocrinol Metab. 1990;71:91822. PMID: 2119392 DOI: $10.1210 /$ jcem-71-4-918 
Griffin D, Feinn R, Engmann L, Nulsen J, Budinetz T, Benadiva C. Dual trigger with gonadotropin-releasing hormone agonist and Standard dose human chorionic gonadotropin to improve oocyte maturity rates. Fertil Steril. 2014;102:405-9. PMID: 24842671 DOI: 10.1016/j.fertnstert.2014.04.028

Humaidan P, Papanikolaou EG, Tarlatzis BC. GnRHa to trigger final oocyte maturation: time to reconsider. Hum Reprod. 2009;24:2389-94. PMID: 19608565 DOI: 10.1093/ humrep/dep246

Jones BP, Al-Chami A, Gonzalez X, Asshad F, Green J, Bracewell-Milnes T, Saso S, Smith R, Serhal P, Nagi JB. Is oocyte maturity influenced by ovulation trigger type in oocyte donation cycles? Hum Fertil. 2019;1:1-7. PMID: 31571498 DOI: $10.1080 / 14647273.2019 .1671614$

Jung YH, Kim YY, Kim MH, Yoo YJ, Jo JD. Optimal usage of dual trigger to prevent HSS in a long protocol IVF cycle. Fertil Steril. 2014;102:e222. DOI: 10.1016/j.fertnstert.2014.07.748

Kim CH, Ahn JW, You RM, Kim SH, Chae HD, Kang BM. Combined administration of gonadotropin-releasing hormone agonist with human chorionic gonadotropin for final oocyte maturation in GnRH antagonist cycles for in vitro fertilization. J Reprod Med. 2014;59:63-8. PMID: 24597289

Kol S. Luteolysis induced by a gonadotropin-releasing hormone agonist is the key to prevention of ovarian hyperstimulation syndrome. Fertil Steril. 2004;81:1-5. PMID: 14711532 DOI: 10.1016/j.fertnstert.2003.05.032

Kol S, Humaidan P. LH (as HCG) and FSH surges for final oocyte maturation: sometimes it takes two to tango? Reprod Biomed Online. 2010;21:590-2. PMID: 20851052 DOI: $10.1016 /$ j.rbmo.2010.06.031

Lin $\mathrm{MH}$, Wu FS, Lee RK, Li SH, Lin SY, Hwu YM. Dual trigger with combination of gonadotropin releasing hormone agonist and human chorionic gonadotropin significantly improves the live birth rate for normal responders in $\mathrm{GnRH}$ antagonist cycles. Fertil Steril. 2013;100:1296 302. PMID: 23993928 DOI: 10.1016/j.fertnstert.2013.07.1976

Lu X, Hong Q, Sun L, Chen Q, Fu Y, Ai A, Lyu Q, Kuang Y. Dual trigger for final oocyte maturation improves the oocyte retrieval rate of suboptimal responders to gonadotropin-releasing hormone agonist. Fertil Steril. 2016;106:1356-62. PMID: 27490046 DOI: 10.1016/j.fertnstert.2016.07.1068

Ludwig M, Doody KJ, Doody KM. Use of recombinant human chorionic gonadotropin in ovulation induction. Fertil Steril. 2003;79:1051-9. PMID: 12738494 DOI: 10.1016/ S0015-0282(03)00173-0

Mahajan N, Sharma S, Arora PR, Gupta S, Rani K, Naidu $P$. Evaluation of dual trigger with gonadotropin-releasing hormone agonist and human chorionic gonadotropin in improving oocyte maturity rates: a prospective randomized study. J Hum Reprod Sci. 2016; 9:101-6. PMID: 27382235 DOI: $10.4103 / 0974-1208.183506$

Nevo O, Eldar-Geva T, Kol BS, Itskovitz-Eldor J. Lower levels of inhibin $A$ and pro-C during the luteal phase after triggering oocyte maturation with a gonadotropin-releasing hormone agonist versus human chorionic gonadotropin. Fertil Steril. 2003;79:1123-8. PMID: 12738506 DOI: 10.1016/S0015-0282(03)00177-8
O’Neill KE, Senapati S, Maina I, Gracia C, Dokras A. GnRH agonist with low-dose hCG (dual trigger) is associated with higher risk of severe ovarian hyperstimulation syndrome compared to GnRH agonist alone. J Assist Reprod Genet. 2016;33:1175-84. PMID: 27349252 DOI: 10.1007/ s10815-016-0755-8

Orvieto R, Zagatsky I, Yullzari-Roll V, La Marca A, Fisch B. Substituting human gonadotropin by gonadotropin releasing hormone to trigger final follicular maturation, during controlled ovarian hyperstimulation, results in less systemic inflammation. Gynecol Endocrinol. 2006;22:437-40. PMID: 17012105 DOI: 10.1080/09513590600881339

Orvieto R. Triggering final follicular maturation - HCG, GnRH agonist or both, when and to whom? J Ovarian Res. 2015;8:60. PMID: 26293447 DOI: 10.1186/s13048-0150187-6

Pacheco FS, Small A, Acosta C, Acosta K, Dunn S, Oktay $\mathrm{K}$. The use of a "double trigger" for fertility preservation in poor responders. Fertil Steril. 2014;102:e163. DOI: 10.1016/j.fertnstert.2014.07.558

Penzias AS. Improving results with assisted reproductive technologies: individualized patient-tailored strategies for ovulation induction. Reprod Biomed Online. 2004;9:43-6. PMID: 15257817 DOI: $10.1016 /$ S1472-6483(10)62108-6

Prochazka R, Blaha M, Nemcova L. Signaling pathways regulating $\mathrm{FSH}-$ and amphiregulin-induced meiotic resumption and cumulus cell expansion in the pig. Reproduction 2012;144:535-46. PMID: 22949725 DOI: 10.1530/ REP-12-0191

Seval MM, Özmen B, Atabekoğlu C, Şükür YE, Şimşir C, Kan Ö, Sönmezer M. Dual trigger with gonadotropin-releasing hormone agonist and recombinant human chorionic gonadotropin improves in vitro fertilization outcome in gonadotropin-releasing hormone antagonist cycles. J Obstet Gynaecol Res. 2016;42:1146-51. PMID: 27199084 DOI: $10.1111 /$ jog. 13021

Schachter M, Friedler S, Ron-El R, Zimmerman AL, Strassburger D, Bern O, Raziel A. Can pregnancy rate be improved in gonadotropin-releasing hormone (GnRH) antagonist cycles by administering GnRH agonist before oocyte retrieval? A prospective, randomized study. Fertil Steril. 2008;90:1087-93. PMID: 18023439 DOI: 10.1016/j.fertnstert.2007.07.1316

Shapiro BS, Daneshmand ST, Garner FC, Aguirre M, Hudson C. Comparison of "triggers" using leuprolide acetate alone or in combination with low-dose human chorionic gonadotropin. Fertil Steril. 2011;95:2715-7. PMID: 21550042 DOI: $10.1016 /$ j.fertnstert.2011.03.109

Simón C, Cano F, Valbuena D, Remohi J, Pellicer A. Clinical evidence for a detrimental effect on uterine receptivity of high serum estradiol concentrations in high and normal responders. Hum Reprod. 1995;10:2432-7. PMID: 8530680 DOI: $10.1093 /$ oxfordjournals.humrep.a136313

Simón C, Garcia Velasco JJ, Valbuena D, Peinado JA, Moreno $C$, Remohí J, Pellicer $A$. Increasing uterine receptivity by decreasing estradiol levels during the preimplantation period in high responders with the use of a follicle stimulating hormone step-down regimen. Fertil Steril. 1998;70:234-9. PMID: 9696213 DOI: 10.1016/S0015-0282(98)00140-X 
Tavaniotou A, Albano C, Smitz J, Devroey P. Impact of ovarian stimulation on corpus luteum function and embryonic implantation. J Reprod Immunol. 2002;55:123-30. PMID: 12062827 DOI: 10.1016/S0165-0378(01)00134-6

Tay CC. Use of gonadotropin-releasing hormone agonist to trigger ovulation. Hum Fertil. 2002;5:G35-7. PMID: 11939159 DOI: $10.1080 / 1464727992000199811$

Valbuena D, Martin J, Palbo JL, Remohi J, Pellicer A, Simón C. Increasing levels of estradiol are deleterious to embryonic implantation because they directly affect the embryo. Fertil Steril. 2001;76:962-8. PMID: 11704118 DOI: 10.1016/S0015-0282(01)02018-0

Veeck LL. Atlas of the human oocyte and early conceptus. Baltimore: Williams \& Wilkings; 1991.
Von Elm E, Altman DG, Egger M, Pocock SJ, Gøtzsche PC, Vandenbroucke JP; STROBE Initiative. The strengthening the reporting of observational studies in epidemiology (STROBE) statement: guidelines for reporting observational studies. Int J Surg. 2014;12:1495-9. PMID: 25046131 DOI: 10.1016/j.ijsu.2014.07.013

Youssef MA, Van Der Veen F, Al-Inany HG, Mochtar MH, Griesinger G, Nagi Mohesen M, Aboulfoutouh I, Van Wely M. Gonadotropin-releasing hormone agonist versus HCG for oocyte triggering in antagonist-assisted reproductive technology. Cochrane Database Syst Rev. 2014;10:CD008046. PMID: 25358904 DOI: 10.1002/14651858.CD008046. pub4 